Dept. of Microbiology,

Faculty of Vet. Med., Al-Baath Univ., Syria.

\title{
ANTIBIOTIC RESISTANCE OF ESCHERICHIA COLI ISOLATED FROM POULTRY IN SYRIA
}

(With 5 Tables)

\section{By \\ B.M. MAKHOL; N. HABREH* and K. SAKURAI**}

* Diagnosis and Laboratory Analysis (Bacterial), Al-Baath University,

Dept. of Microbiology, Faculty of Vet Med, Syria.

**A volunteer expert from JICA (The Japan International Corporation Agency)

(Received at 10/11/2010)

\section{مقاومة المضادات الحيوية للإشريكية القولونية المعزولة من الاواجن في سوريا بركات ميشيل مخول ، ناجح هبره ، كينيتشي ساكوراي}

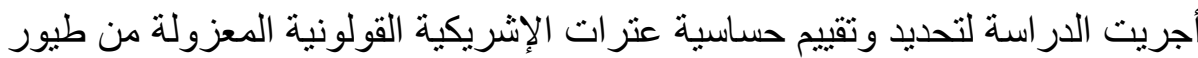

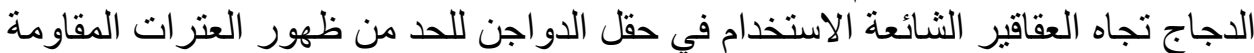

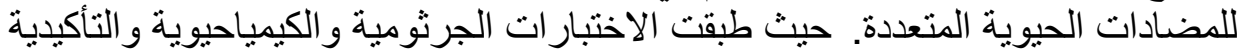

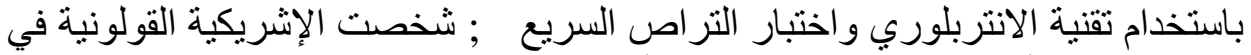

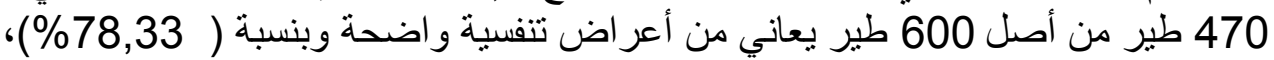
حيث تم عزل 1060 عزولة من الإشريكية القولونية من الأحشاء الداخلية (قلب، رئنتين،

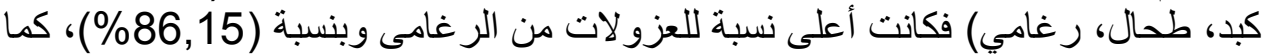

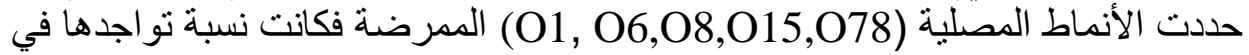
العزو لات (42,55\%). كما أظهرت نتائج هذه الدراسة أن كل العزو لات المختبرة كانت

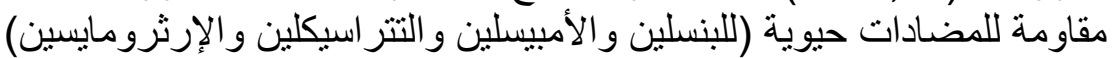
بنسبة (100\%)، وكانت بنسبة (95,7\%) و (91,4\%) من العزو لات مقاومة للكنامايسين و النيو مايسين على التو الي. بينما كانت حساسة للكوليستين بنسبة (

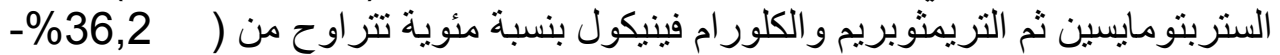

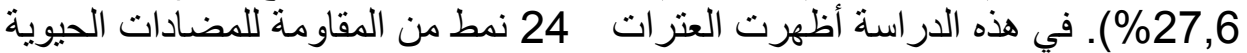
المختلفة (11:وب ع عن المضادات الحيوية).

\section{SUMMARY}

The study was carried out to define and evaluate the sensitivity of E.coli strains isolated from poultry to common antibiotics used in the field in order to limit the appearance of multi-antibiotic-resistant strains. Then after applying the bacterial or germinal and conformational biochemical tests by using intropolory techniques and quick agglutination tests. Existence of E.coli was diagnosed in 470 out of 600 hens suffering clear respiratory 
symptoms (78.33\%). A number of 1060 isolates of E.coli had been taken from internal viscera, namely hearts, lungs, livers, spleens, tracheas. The latter contributed the highest percentage $(86.15 \%)$ of the total number of isolates. Moreover, five patterns of pathogenic serum of E.coli $(\mathrm{O} 1, \mathrm{O} 6, \mathrm{O} 8, \mathrm{O} 15, \mathrm{O} 78)$ were identified in $(42.55 \%)$ of the isolates. The research results demonstrated that $(100 \%)$ of all tested isolates were resistant to certain antibiotics (Penicillin, Ampicyllin, Tetracycline and Erythromycin) where $95.7 \%$ and $91.4 \%$ of the isolates were resistant to Kanamycin and Neomycin, respectively. On the other hand, (69.4\%) of the isolates were found to be sensitive to Colistin, while sensitivity to Streptomycin, Trimethoprim and Chloramphenycol was between $(36.2 \%$ $27.6 \%$ ). Finally speaking, in this study, E.coli strains were found to exhibit 24 resistance patterns to 11 different types of antibiotics.

Key words: Poultry, E.coli, antibiogram.

\section{INTRODUCTION}

\section{المقدمـة}

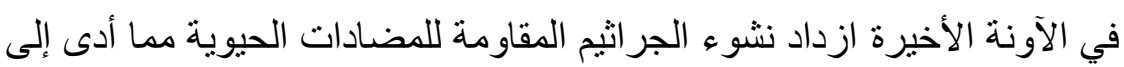

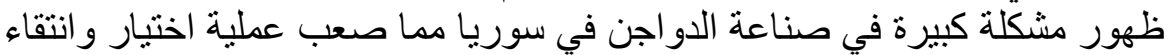
المضادات الحيوية الفعالة في معالجة الحالات المرضية المئة المختلفة في الحقل، هذه المشكلة

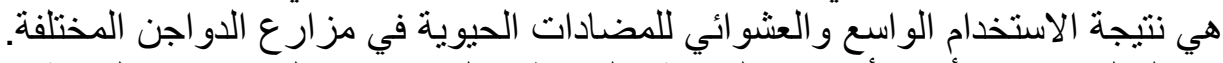

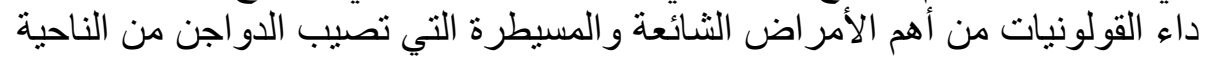
الاقتصادية (Margie et al., 1999). الإشريكية القولونية هي المسبب الرئيسي لهذا المرض و المسؤولة عن العديد من

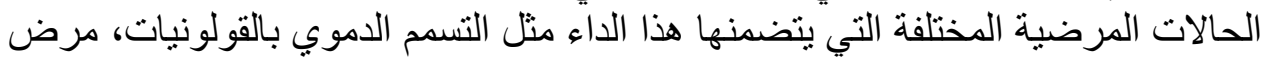

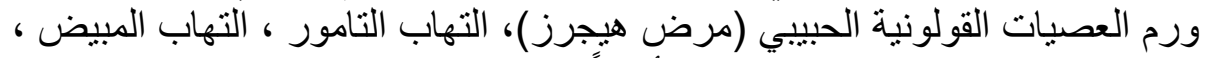

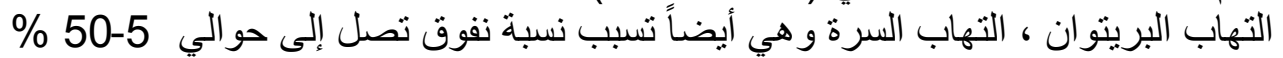

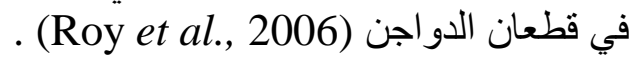

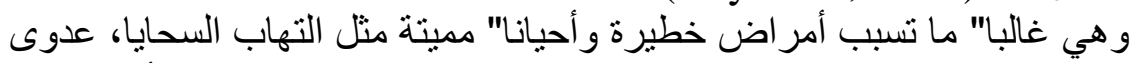

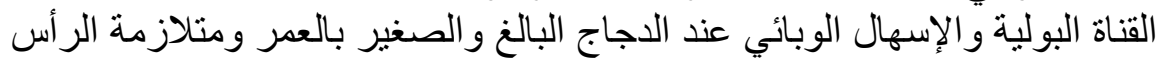

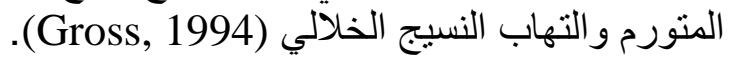

فالإشريكية القولونية هي إحدى المسببات المرضية الأكثر شيو عاً وانتشاراً بين

الجر اثيم سلبيـة الغرام في إحداث الأمرا ض الثنائعـة التي تصيب الدواجن Diekema) .et al., 1999)

على الرغم من أن ظاهرة مقاومة المضادات الحيوية من قبل الجر اثثم سلبية

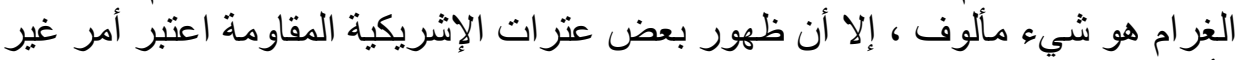

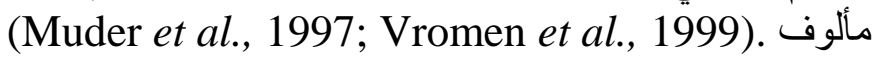




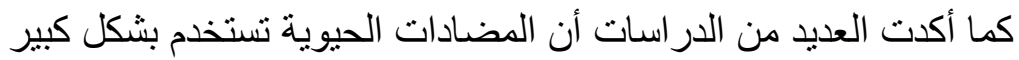

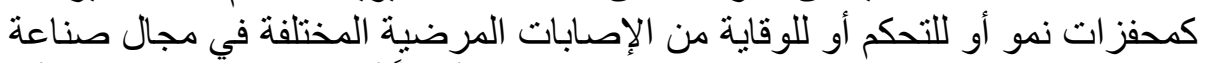

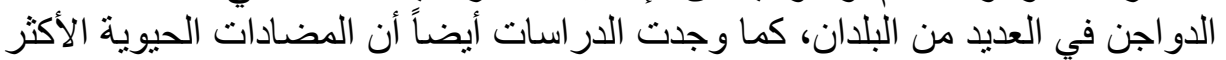

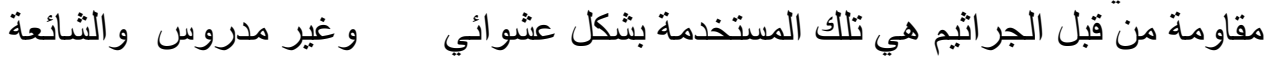

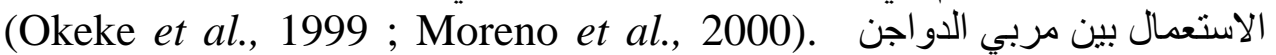

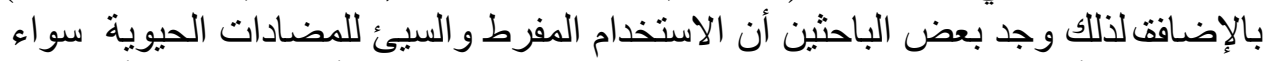

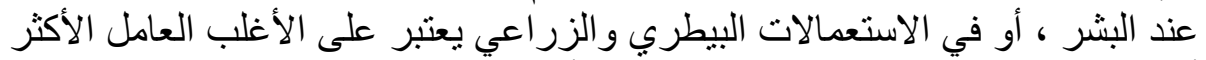

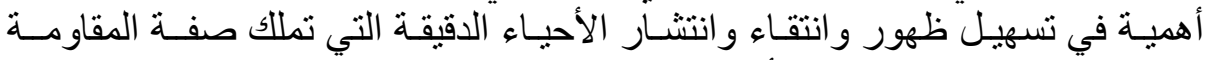

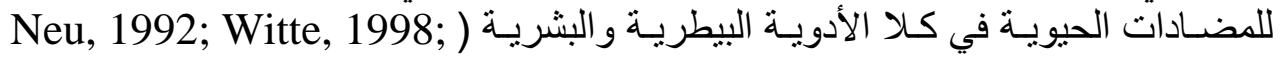
(Gunner et al., 2004

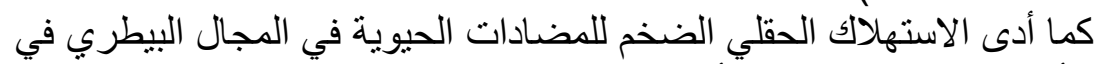

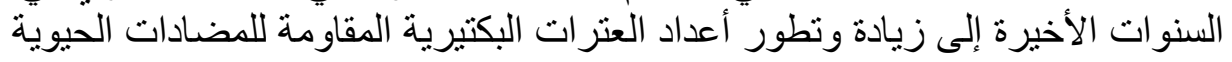

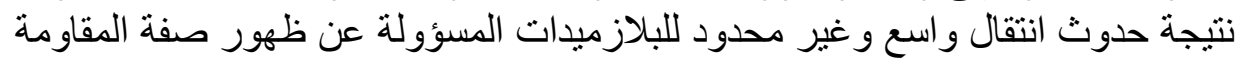

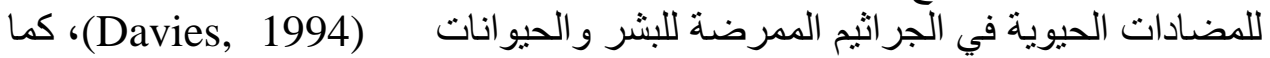

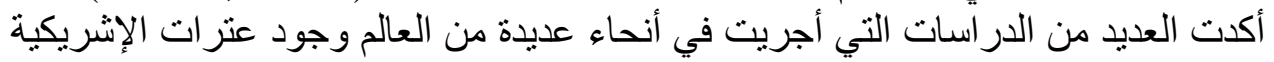

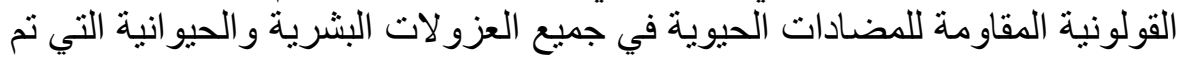
دراستها (Amara et al., 1995). توجد تقارير عن مقاومة الإشريكية للمضادات الحيوية المتر افقة مع فثنل المعالجة

.(Talan et al., 2004; Blondeau, 2004)

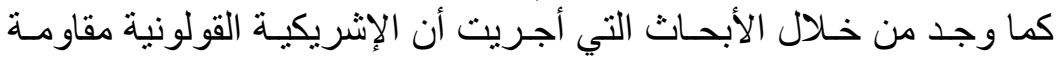

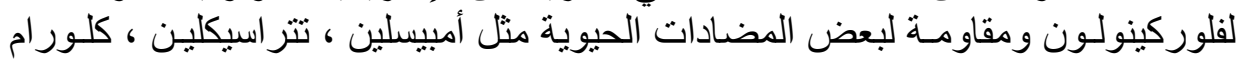

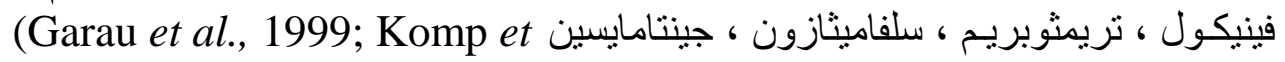
كمـا وجد أيضـا أن هنـالك زيادة هامة في مقاومة الإشريكيـة القولونيـة .al., 2003)

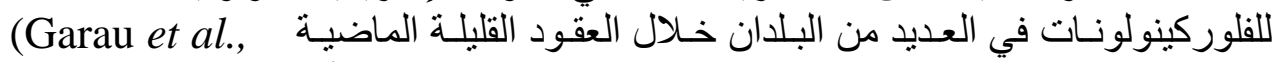
(أدرجت في قائمسة من : 1999; Van Blkum et al., 2001; Viroy et al., 2005)

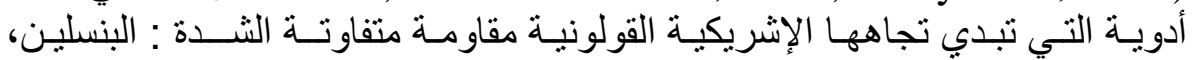

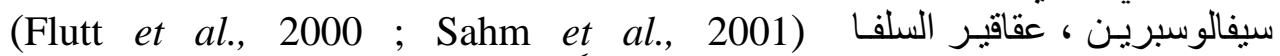

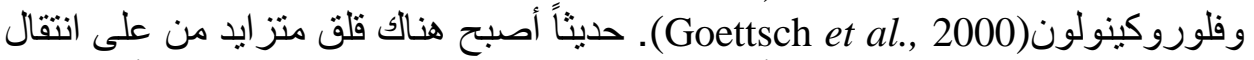

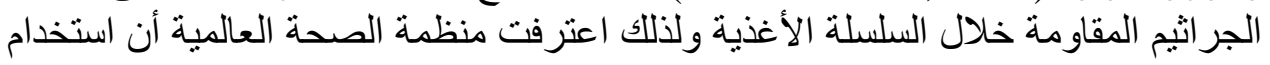

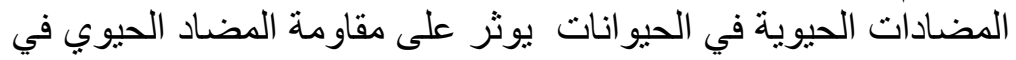
الإنسان (Anonymous, 2000).

$$
\text { الغرض من البحث: الإن }
$$

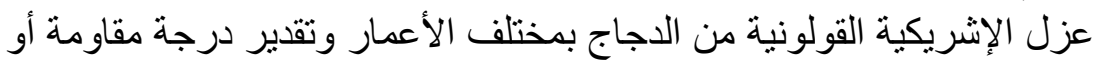
حساسية العتر ات المعزولة لبعض الصنادات الحيوية المستعملة في المجال الحقلي.

\section{MATERIALS and METHODS}


المواد المستخدمة في البحث وطرق العمل

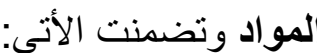

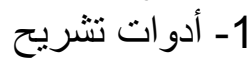

2- 2- حافظات تحوي مبردات لحفظ العينات

3- 3- وسط قاعدة الاجار الدذمى

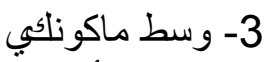

4- 5- وسط الأيوزين وازرق المثيلين (EMB)

5- كو اثف الاومو اد لتحضير الاختبار ات الكيمياحيوية

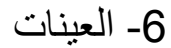

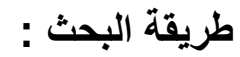

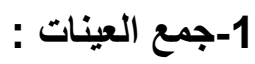

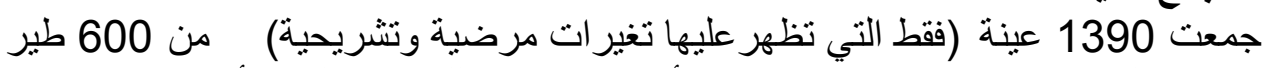

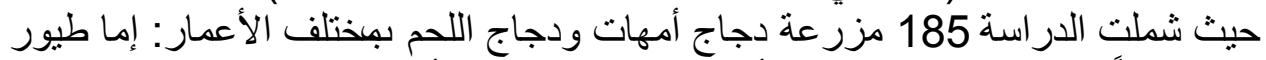

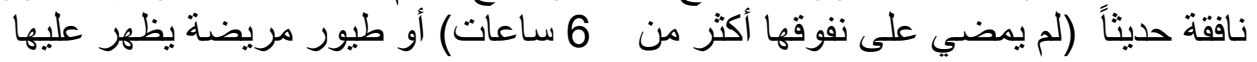

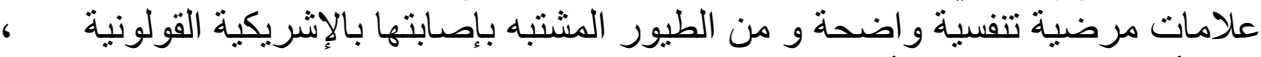

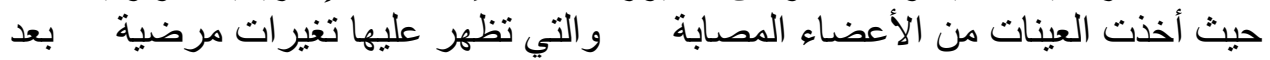

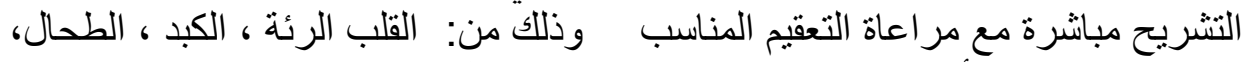

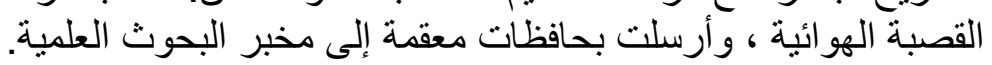

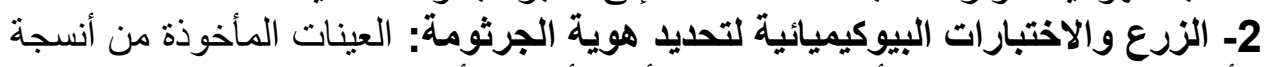

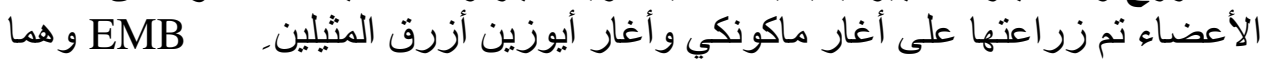

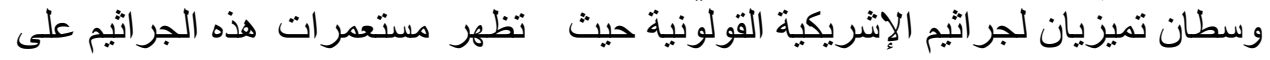

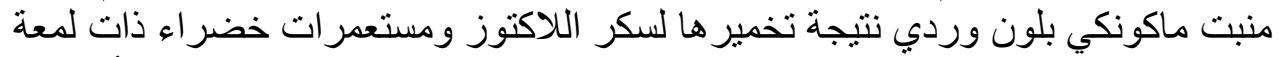

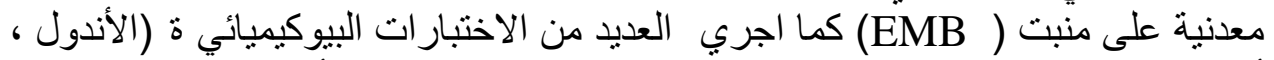

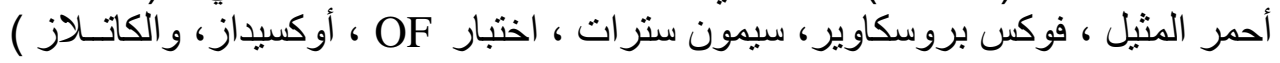

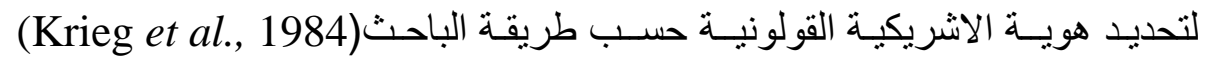

الجدول 1: نتائج الاختبارات البيوكيميائية لتحديد هوية عزولات الإشريكية القولونية

\begin{tabular}{|c|c|c|c|c|c|c|c|c|c|}
\hline عتر ات & غر ام & الشكل & $\mathrm{OF}^{*}$ & الكاتالاز & الأوكسيداز & الأندول & $\mathrm{MR}^{* *}$ & $\mathrm{VP} * * *$ & الستر ات \\
\hline 1060 & - & عصوي & + & + & - & + & + & - & - \\
\hline
\end{tabular}

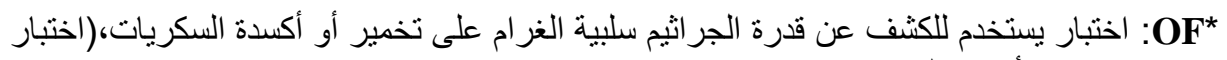

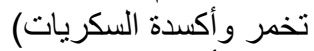

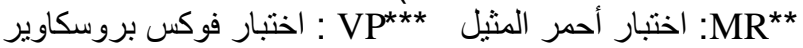

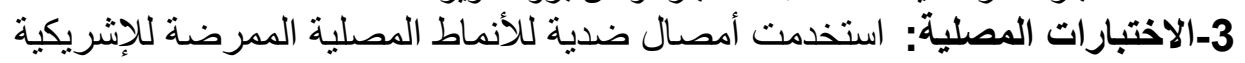

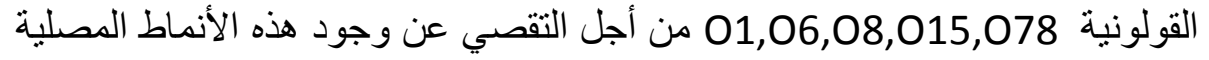
للإنثريكية القولونية الممرضة الطيرية ضمن هذه العزولات، حسب الطرق القيانية 
للباحثين (Glantz et al., 1962 ; Orskov et al., 1977) و الموصوفة من قبل شركة Denka Seiken Co.Ltd, Tokyo, Japan

4- اختبارات الحساسية: أجريت هذه الاختبار ات بطريقة الانتشار بهدف اختيار

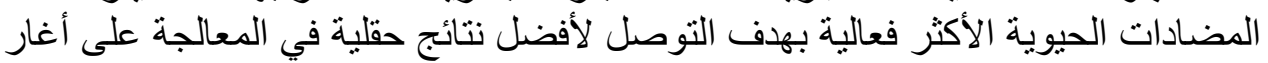

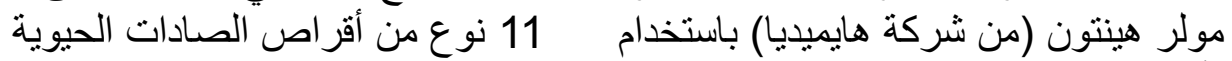

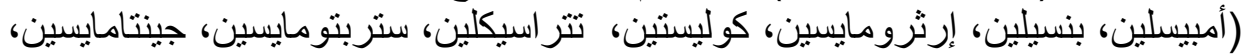

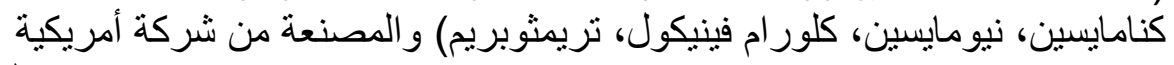
(Becton Dickinson Microbiology Company,USA) طريقة (Bauer et al., 1966).

\section{RESULTS}

\section{النتائسج}

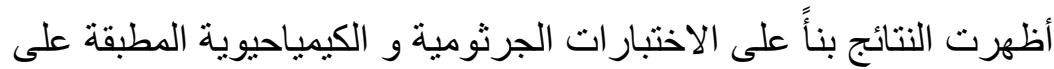

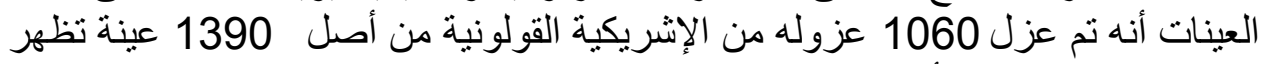

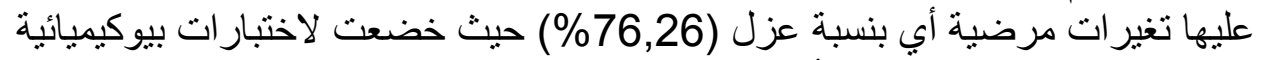
وشكلية لتحديد هويتها ، فكانت أعلى نسبة لتو اجد هذه الجر اثيم في الرغامى ( و أقلها في الكبد (70,18\%).

جدول رقم 2: يوضح توزع العزو لات ومعدلات الإصابة حسب الأعضاء المدروسة

\begin{tabular}{|c|c|c|c|}
\hline معدل الإصابة\%\% & عزو لات الإشريكية & عدد الأعضاء & العضو المفحوص \\
\hline 75,60 & 220 & 291 & القلب القب \\
\hline 75,54 & 210 & 278 & الرئتين \\
\hline 70,18 & 200 & 285 & الكبد \\
\hline 71,09 & 150 & 211 & الطحال \\
\hline 86,15 & 280 & 325 & القصبة الهوائية \\
\hline 76,26 & 1060 & 1390 & المجموع \\
\hline
\end{tabular}

وبإجر اء التنميط المصلي لعزو لات الاشريكية القولونية للتقصي عن الأنماط

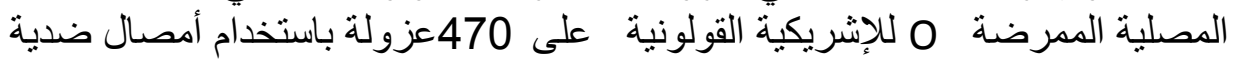

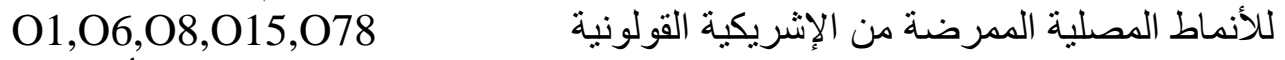
[DENKA SEIKEN Co.Ltd,Tokyo,Japan] (42.55\%) موزعة على الثكل النالي : النمط المصلي و المسيطر في هذه الدراسة وبنسبة تو اجد (

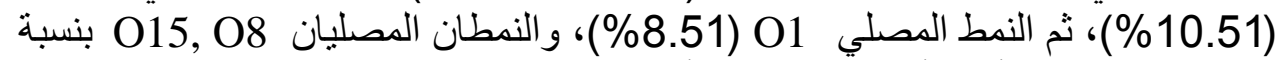

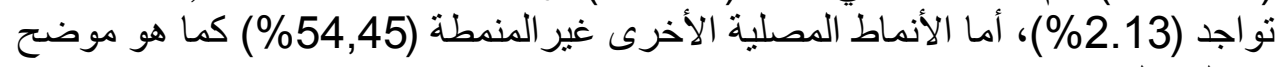
في الجدول (3). (2) - (2) 
الجدول 3: تتمبط عزو لات الإشريكية القولونية التي نم عزلها في هذه الدر اسة الدية

\begin{tabular}{|c|c|c|}
\hline النسبة المئوية للانماط المصلية O O & عدد الأنماط المصلية O المعزولة & الأنماط المصلية \\
\hline 8,51 & 40 & $\mathrm{O} 1$ \\
\hline 10,64 & 50 & O6 \\
\hline 2,13 & 10 & O8 \\
\hline 2,13 & 10 & O15 \\
\hline 19,15 & 90 & $\mathrm{O} 78$ \\
\hline 42,55 & 200 & الإجمالى \\
\hline
\end{tabular}

أظهرت عزو لات الإشريكية القولونية (1000 عزولة تم اختبار درجة تحسسها

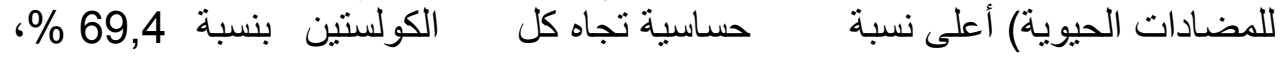

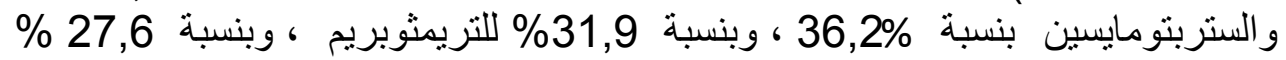
للكلور ام فينيكول وبنسبة 12,7 \% \% للجنتامايسين أما النيو ماسين و الكنامايسين فكانت نسبة التحسس لكل منهما متساوية 2,1 \% \%. بينما أبدت أغلب العزو لات مقاو مة متفاوتة الثندة

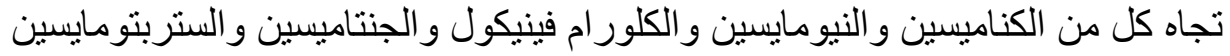

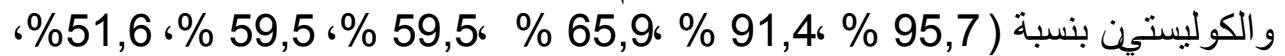

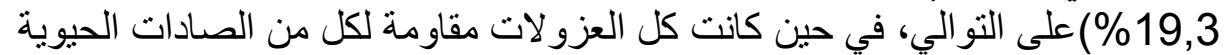

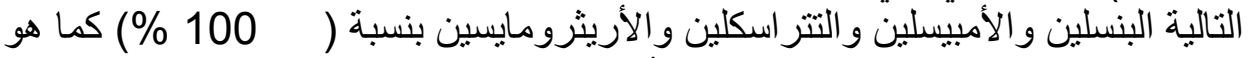

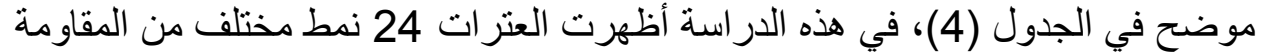

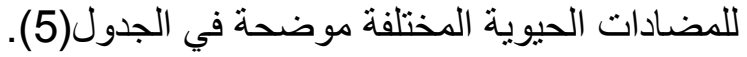
الجدول 4: نسبة نأثنير المضادات الحيوية على عزو لات الإشريكية القولونية

\begin{tabular}{|c|c|c|c|}
\hline \multicolumn{3}{|c|}{ النسبة المئوية للعزولات \% } & المضساد الحيوي \\
\hline الحساسة & متوسطة الحساسية & المقاومة & درجة المقاومة \\
\hline 0 & 0 & 100 & بنسلين \\
\hline 0 & 0 & 100 & أمبيسلين \\
\hline 0 & 0 & 100 & 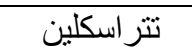 \\
\hline 69,4 & 11,3 & 19,3 & كوليستين \\
\hline 0 & 0 & 100 & إرثرومايسين \\
\hline 36,2 & 12,2 & 51,6 & ستربيو مايسين \\
\hline 12,7 & 27,6 & 59,7 & جنتامايسين \\
\hline 2,1 & 6,5 & 91,4 & نيومايسين \\
\hline 2,1 & 2,2 & 95,7 & كنامايسين \\
\hline 27,6 & 6,5 & 65,9 & كلور امفينيكول \\
\hline 31,9 & 8,6 & 59,5 & تريمثوبريم \\
\hline
\end{tabular}

الجدول 5: مقاومة الصنادات الحيوية المتعددة لعزو لات الإشريكية القولوني المدروسة

\begin{tabular}{|c|c|c|c|c|c|c|c|c|c|c|c|c|c|c|}
\hline المضادات & المقوذج مة & \multicolumn{11}{|c|}{ نماذج مقاومة المضادات الحيوية من قبل الإشريكية القولونية } & \multicolumn{2}{|c|}{ 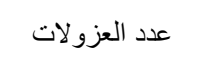 } \\
\hline & & PC & AM & GM & KM & SM & EM & $\mathrm{CL}$ & TC & C & NM & STX & التكر ار & الإجمالي \\
\hline & 1 & $R$ & $R$ & $R$ & $R$ & $R$ & $R$ & - & $R$ & $\mathrm{R}$ & $R$ & $R$ & 185 & \\
\hline
\end{tabular}




\begin{tabular}{|c|c|c|c|c|c|c|c|c|c|c|c|c|c|c|}
\hline \multirow[t]{2}{*}{10} & 2 & $\mathrm{R}$ & $\mathrm{R}$ & - & $\mathrm{R}$ & $\mathrm{R}$ & $\mathrm{R}$ & $R$ & $\mathrm{R}$ & $\mathrm{R}$ & $R$ & $\mathrm{R}$ & 42 & \multirow[t]{2}{*}{250} \\
\hline & 3 & $\mathrm{R}$ & $\mathrm{R}$ & $R$ & $R$ & $R$ & $\mathrm{R}$ & $R$ & $\mathrm{R}$ & $R$ & - & $\mathrm{R}$ & 23 & \\
\hline \multirow{5}{*}{9} & 4 & $R$ & $\mathrm{R}$ & - & $\mathrm{R}$ & $\mathrm{R}$ & $R$ & - & $R$ & $R$ & $\mathrm{R}$ & $R$ & 82 & \multirow{5}{*}{310} \\
\hline & 5 & $\mathrm{R}$ & $\mathrm{R}$ & $\mathrm{R}$ & $\mathrm{R}$ & - & $\mathrm{R}$ & - & $\mathrm{R}$ & $\mathrm{R}$ & $\mathrm{R}$ & $\mathrm{R}$ & 122 & \\
\hline & 6 & $\mathrm{R}$ & $\mathrm{R}$ & $R$ & $\mathrm{R}$ & $\mathrm{R}$ & $\mathrm{R}$ & - & - & $\mathrm{R}$ & $\mathrm{R}$ & $\mathrm{R}$ & 23 & \\
\hline & 7 & $\mathrm{R}$ & $\mathrm{R}$ & $\mathrm{R}$ & $\mathrm{R}$ & $\mathrm{R}$ & $\mathrm{R}$ & - & $\mathrm{R}$ & - & $\mathrm{R}$ & $\mathrm{R}$ & 63 & \\
\hline & 8 & $\mathrm{R}$ & $\mathrm{R}$ & - & $\mathrm{R}$ & $\mathrm{R}$ & $R$ & $R$ & - & $R$ & $\mathrm{R}$ & $\mathrm{R}$ & 20 & \\
\hline \multirow{8}{*}{8} & 9 & $\mathrm{R}$ & $\mathrm{R}$ & $\mathrm{R}$ & - & $\mathrm{R}$ & $\mathrm{R}$ & - & - & $\mathrm{R}$ & $R$ & $\mathrm{R}$ & 22 & \multirow{8}{*}{190} \\
\hline & 10 & $R$ & $R$ & - & $\mathrm{R}$ & - & $\mathrm{R}$ & $R$ & $\mathrm{R}$ & $\mathrm{R}$ & - & $\mathrm{R}$ & 21 & \\
\hline & 11 & $R$ & $\mathrm{R}$ & - & $\mathrm{R}$ & - & $\mathrm{R}$ & - & $\mathrm{R}$ & $\mathrm{R}$ & $\mathrm{R}$ & $\mathrm{R}$ & 23 & \\
\hline & 12 & $\mathrm{R}$ & $\mathrm{R}$ & $\mathrm{R}$ & $\mathrm{R}$ & - & $R$ & - & $\mathrm{R}$ & - & $\mathrm{R}$ & $\mathrm{R}$ & 24 & \\
\hline & 13 & $R$ & $\mathrm{R}$ & - & $\mathrm{R}$ & $R$ & $\mathrm{R}$ & $R$ & - & - & $R$ & $\mathrm{R}$ & 20 & \\
\hline & 14 & $\mathrm{R}$ & $\mathrm{R}$ & - & $\mathrm{R}$ & $\mathrm{R}$ & $\mathrm{R}$ & - & - & $R$ & $\mathrm{R}$ & $\mathrm{R}$ & 20 & \\
\hline & 15 & $\mathrm{R}$ & $\mathrm{R}$ & $\mathrm{R}$ & $R$ & - & $R$ & $\mathrm{R}$ & - & - & $\mathrm{R}$ & $R$ & 40 & \\
\hline & 16 & $\mathrm{R}$ & $\mathrm{R}$ & $\mathrm{R}$ & $\mathrm{R}$ & - & $\mathrm{R}$ & - & - & $\mathrm{R}$ & $\mathrm{R}$ & $\mathrm{R}$ & 20 & \\
\hline \multirow{6}{*}{7} & 17 & $R$ & $\mathrm{R}$ & $\mathrm{R}$ & $\mathrm{R}$ & - & $\mathrm{R}$ & - & - & - & $R$ & $R$ & 41 & \multirow{6}{*}{150} \\
\hline & 18 & $R$ & $\mathrm{R}$ & - & $\mathrm{R}$ & - & $\mathrm{R}$ & $R$ & - & - & $R$ & $\mathrm{R}$ & 21 & \\
\hline & 19 & $R$ & $R$ & $\mathrm{R}$ & - & - & $\mathrm{R}$ & - & $\mathrm{R}$ & $\mathrm{R}$ & - & $\mathrm{R}$ & 23 & \\
\hline & 20 & $\mathrm{R}$ & $\mathrm{R}$ & - & $\mathrm{R}$ & $\mathrm{R}$ & $\mathrm{R}$ & - & - & - & $\mathrm{R}$ & $R$ & 20 & \\
\hline & 21 & $\mathrm{R}$ & $\mathrm{R}$ & - & $\mathrm{R}$ & - & $\mathrm{R}$ & - & $\mathrm{R}$ & - & $\mathrm{R}$ & $\mathrm{R}$ & 23 & \\
\hline & 22 & $R$ & $\mathrm{R}$ & - & $R$ & - & $\mathrm{R}$ & - & - & $R$ & $R$ & $\mathrm{R}$ & 22 & \\
\hline 6 & 23 & $R$ & $\mathrm{R}$ & - & $R$ & - & $\mathrm{R}$ & - & - & - & $R$ & $\mathrm{R}$ & 70 & 70 \\
\hline 5 & 24 & $R$ & $\bar{R}$ & - & $\bar{R}$ & - & $R$ & - & - & - & - & $\mathrm{R}$ & 30 & 30 \\
\hline الإجمالي & 24 & & & & & & & & & & & & & 1000 \\
\hline
\end{tabular}

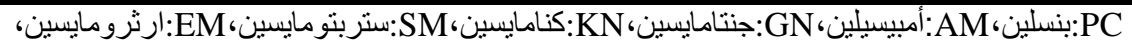

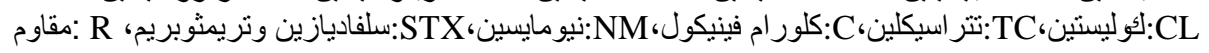

\section{DISCUSSION}

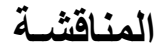

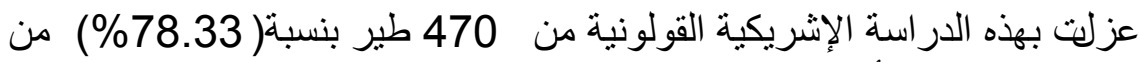

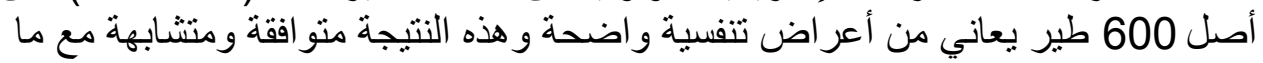
سجله الباحث (Sharada et al., 2010) حيث كانت نسبة عزل للإنشريكية القولونية

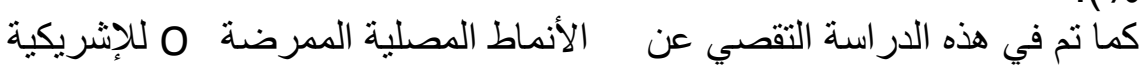
. $(\% 76,47)$

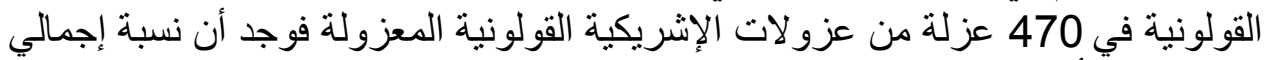

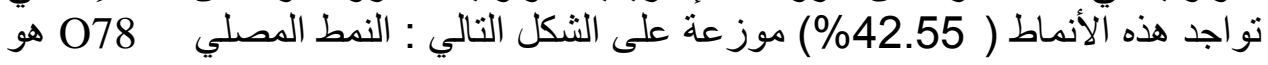
النمط السائد و المسيطر في هذه الدراسة بنسبة تواجد (10.151\%)

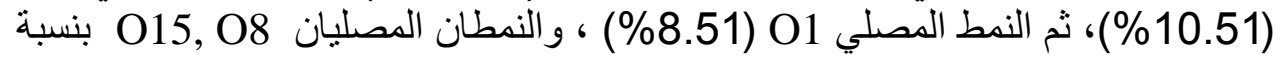




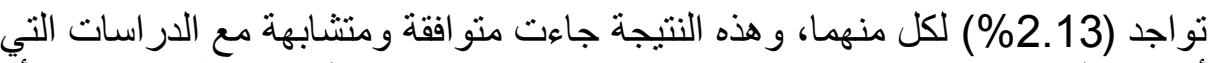

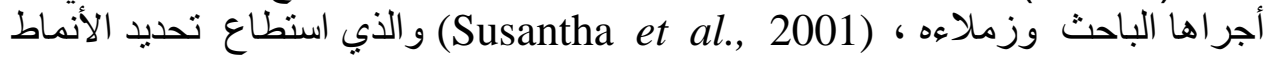
المصلية O8 O81,O2, O78 من حالات مصابة بداء القولونيات الطيري في كندا.

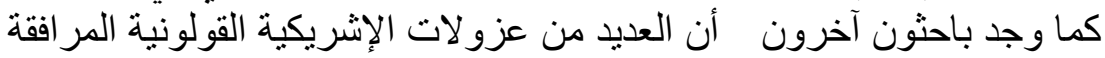

بشكل شائع لداء القولونيات الطيرن آخرن تنتمي في أغلب الأحيان للأنماط الميكية المصلية الممرضة ‘(Sojka and Carnagham,1961; Dho-Moulin et al., 1999) O1,O2, O78 و هذا أيضاً منو افقة مع ما تم إيجاده في هذه الدر اسة.

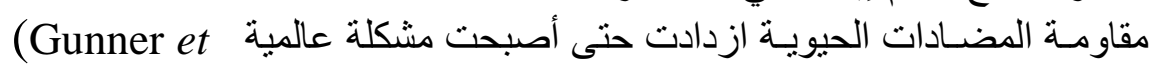
و al., 2004)

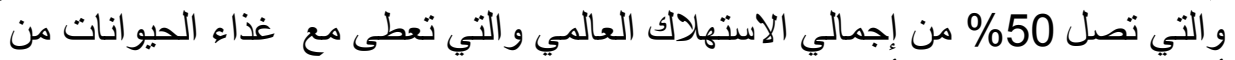

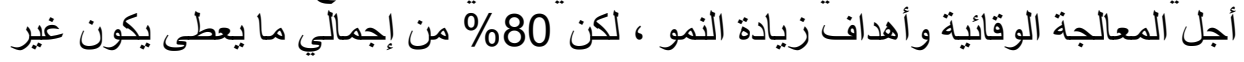

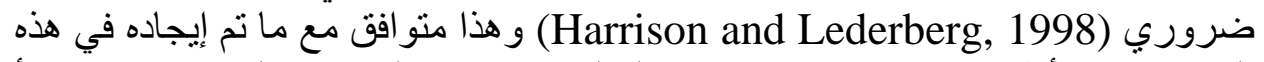

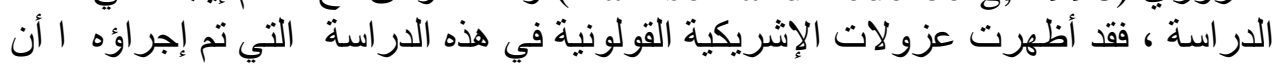

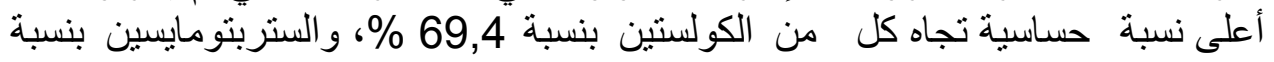

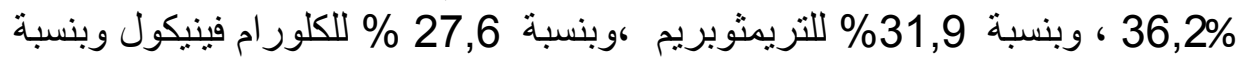

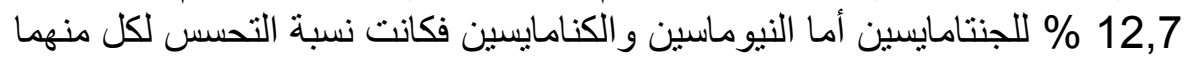

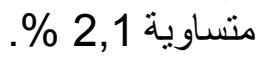
بينما أبدات أغلب العزو لات مقاومة متفاوتة الثدة تجاه كل الكناميسين

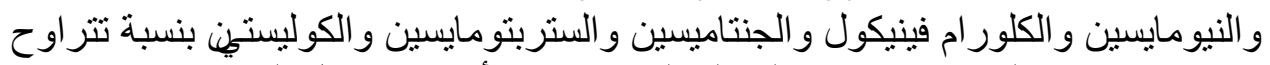

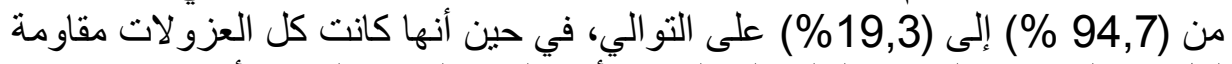

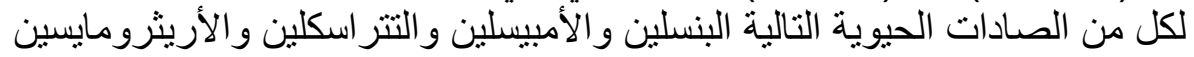

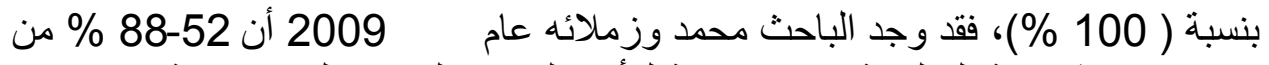

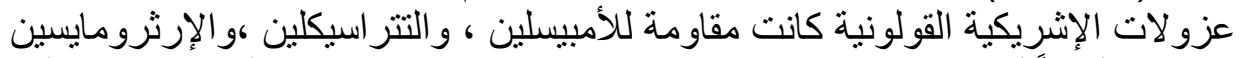

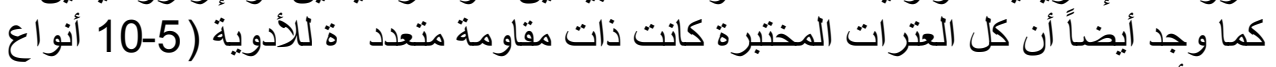

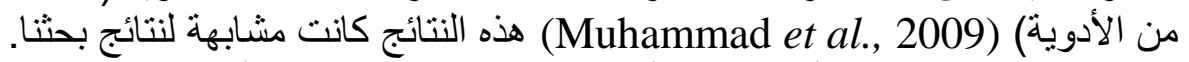

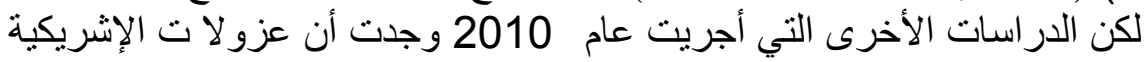

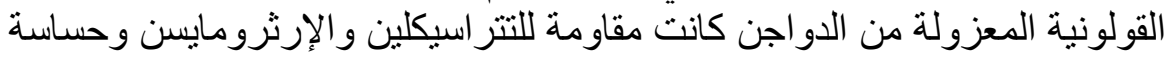

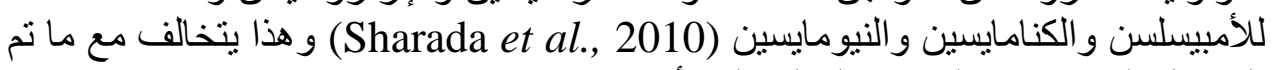
التوصل إليه في هذه الدراسة الحالية التي أجريت.

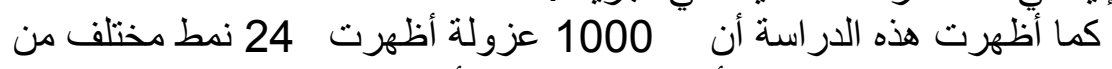

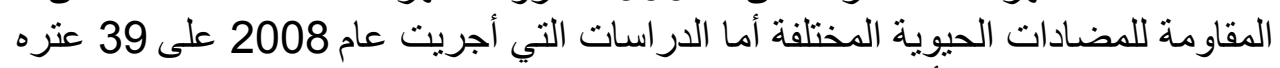
من الإثريكية القولونية أظهرت 19 نمط مختلف من المقاومة للمضادئ المرادات الحيوية المختلفة .(Akinlabi et al., 2008)

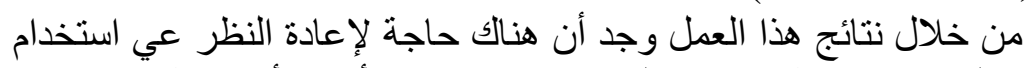

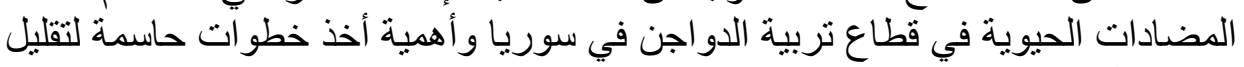

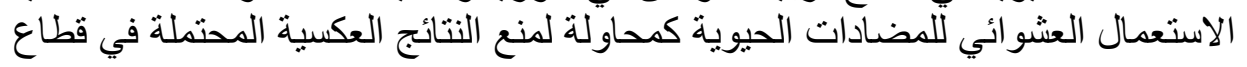




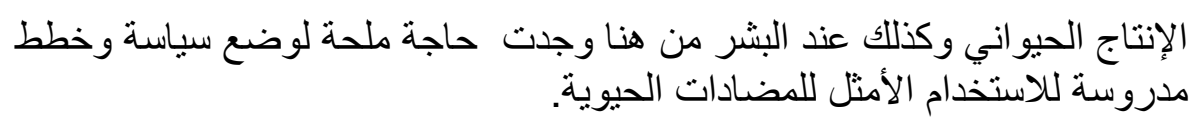

\section{REFERENCES}

Amara, A.; Ziani, Z. and Bouzoubaa, K. (1995): Antibiotic resistance of Escherichia colistrains isolated in Morocco from chickens with colibacillosis.Vet. Microbiol., 43: 325-330.

Anonymous (2000): WHO global principles for containments of antimicrobial resistance in animals intended for food. Geneva: WHO/CDS/CSR/APH/2001.4.

Bauer, A.W.; Kirby, W.M.M.; Sheris, J.C. and Truck, M. (1966): Antibiotic susceptibility testing by a standardized single disc method. American J. Clinical Pathol., 145: 225-230.

Blondeau, J.M. (2004): Current issues in the management of urinary tract infections: extending release ciprofloxacin as a novel treatment options. Drugs 64: 611-628.

Davies, J. (1994): Inactivation of antibiotics and the dissemination of resistance genes. Science, 264: 375-382.

Dho, M. and Lafont, J.P. (1984): Adhesive properties and iron uptake ability in Escherichia coli lethal and non-lethal for chicks. Avian Diseases. 26: 787-797.

Diekema, D.J.; Pfaller, M.A.; Jones, R.M.; Doern, G.V.; Winokur, P.L. and Gales, A.C. (1999): Survey of blood stream infection due to gram negative bacilli frequency of occurrence and antimicrobial susceptibility of isolates collected in the United States, Canada and Latin America for the Sentry Antimicrobial Surveillance Program, 1997. Clin. Infect. Dis. 29: 595-607.

Flutt, A.C.; Jone, M.E.; Schmitz, F.J.; Acar, J.; Guptes, R. and Verhoef, J. (2000): Antimicrobial susceptibility and frequency of occurrence of clinical blood isolate in Europe from Sentry Antimicrobial Surveillance Program 1997 and 1998. Clin. Infect. Dis. 30: 454-460.

Garau, T.; Xercavins, M.; Rodriguez-Carhalleira, M.; Gomez-Vera, J.R.; Coll, I. and Vidal, D. (1999): Emergence and dissemination of quinolone resistant Escherichia coli in the community. Antimicrob. Agents Chemother. 43: 2736-2741.

Goettsch, W.; Van Pelt, W.; Nagelkerke, N.; Hendrix, M.G.; Buiting, A.G. and Petit, P.L. (2000): Increasing resistance to fluoroquinolones in Escherichia coli from urinary tract infections in the Netherlands. JAntimicrob. Chemother. 46: 223-228. 
Gross, W.B. (1994): Diseases due to Escherichia coli in poultry. In C. L. Gyles (ed.). Escherichia coli in Domestic Animals and Humans. CAB International . Wallingford, UK, 237-260.

Gunner, S.S.; John, W.L.; Benedetta, A.; Elizabeth, A.L. and Stefano, L. (2004): The antimicrobial resistance containment and surveillance approach - a public health tool. WHO Bulletin 82: 12.

Harrison, P.I. and Lederberg, I. (1998): Antimicrobial resistance: issues and options. Washington D.C, National Academy Press.

Kreig, N.R.; Holt, J.G. and Williams and Wilkins, (1984): Bergeys Manual of Systematic Bacteriol.,1:428, East Preston street, Baltimore, M.D.2 1202, USA.

Margie, D.L. and Lawrence, H.A. (1999): A Laboratory manual for the isolation and identification of avian pathogenic. $4^{\text {th }}$ ed. American Association of Avian Pathogenic, Athens, G A.

Moreno, M.A.; Dominguez, L.; Teshoger, T.; Herrero, I.A. and Porrero, M.E. (2000): Antibiotic resistances monitoring: The Spanish Program me. Int. J.Antimicrob. Agents., 14: 285-290. DOI: 10. 1016/S0924-8579(00)00138-2

Muder, R.R.; Brennen, C.; Drenning, S.D.; Stout, J.E. and Wagener, M.M. (1997): Multiple antibiotic resistant gram negative bacilli in a long term care facility: a case control study of patient risk factor and prior antibiotic use. Infect. Control Hosp. Epidemiol. 18: 809-813.

Muhammad Ali Akond; Hassan, S.M.R.; Saidul Alam and Momena Shirin, (2009): Antibiotic Resistance of Escherichia coli Isolated from Poultry and Poultry Environment of Bangladesh . Amerixan Journal of Environmental Sciences. 5(1): 47-52

$\mathrm{Neu}$, H.C. (1992): The crisis in antibiotic resistance. Science 257: 1064-1073.

Okeke, I.N.; Lamikanra, A. and Edelman, R. (1999): Socio-economic and behavioral factors leading to acquired bacterial resistance to antibiotics in developing countries. Emerg. Infect. Dis., 5: 13-27.

Roy, P.; Edwin, P.G. and Purushothaman, V. (2006): Isolation of Escherichia coli isolates from hatchery and breeder hen. Indian Veterinary J., 87: 75-82.

Sharada, S.; Wilfred Ruban and Thiyageeswaran, M. (2010): Isolation, characterization and antibiotic pattern of Escherichia coli isolated from poultry. Amer-Euro J. Sci Res5(1):18-22.

Sahm, D.F.; Thornsberry, C.; Mayfield, D.C.; Jones, M.E. and Karlowsky, J.A. (2001): Multidrug resistant urinary tract isolates of 
Escherichia coli prevalence and patient dermographic in United States in 2000. Antimicrob. Agents Chemother. 45: 1402-1406.

Sojka, W.J. and Carnagham, R.B.A. (1961): E. coli in poultry. Research in Veterinary Science. 2: 340-351.

Susantha M. Gomis; Craig Riddell; Andrew, A.; Potter, Brenda and Allan, $J$. (2001): Phenotype and genotypic characterization of virulence facters of Escherichia coli isolated from broiler with simultaneous occurrence of cellulitis and other colibacillosis lesions. Can J. Vet. Res, 65: 1-6.

Talan, D.A.; Naber, K.G.; and Palou, Elkharrat, J.D. (2004): Extended release ciprofloxacin (cipro XR) for treatment of urinary tract infections. Int. J. Antimicrob. Agents 23: 554-566.

Van Belkum, A.; Goessens, W.; Van Der Schee, C.; Lemmens-Dens Toom, N.; Vos, M.C. and Cornelissen, J. (2001): Emergence of ciprofloxacin resistant enterobacteriaea containing multiple gentamycin resistant associated integrons in a Dutch hospital. Emerg. Infect. Dis. 7: 862-871.

Viroy, M.; Lunkin, D.; Maslow, J.N.; Stieritz, D.D.; Carson, L.S. and Bilker, W.B. (2005): Longitudinal trends in antimicrobial susceptibilities across long term care facilities emergence of fluoroquinolone resistance. Infect. Control. Hosp. Epidemiol. 26: 56-62.

Vromen, M.; Vander Ven, A.M.; Knols, A. and Stobberingh, E.E. (1999): Antimicrobial resistance pattern in urinary isolates from nursing home residents: fifteen years of data review. J. Antimicrob. Chemother. 44: 113-116.

Witte, W. (1998): Medical consequences of antibiotics use in Agriculture. Science 279: 996-997. 